\title{
Hybrid Renewable Energy System Integration with Grid - A Review
}

\author{
Aftab Alam \\ M. Tech Scholar \\ Bhabha College of Engineering RKDF University \\ Bhopal, Madhya Pradesh, India \\ aftabalam1996786@gmail.com
}

\author{
Md. Varsha Mehar \\ Assistant Professor \\ Bhabha College of Engineering RKDF University \\ Bhopal, Madhya Pradesh, India
}

\begin{abstract}
In contrast to a framework rely on a specific source, hybrid power system systems (HESs) integrate various initiating, stockpiling, and consuming methodologies into a single system, enhancing overall benefits. Originally intended encompass long established, depletable generation (e.g., diesel generators) and battery storage (BESSs), their definition was been expanded encompass mechanisms that are completely fueled by renewable energy [e.g., solar photovoltaics (PV) and wind], as well as constructions that combine different energy storage systems. This paper provides the systematic literature review relying on the usage of differential evolutionary algorithm to maintain the load demand in hybrid energy systems.
\end{abstract}

Keywords: Hybrid Energy Systems, Distributed Generators, Microgrids, Differential Evolutionary Algorithm

\section{INTRODUCTION}

The majority of electrical components makes use of power sources. Their apps span a wide range of product categories, from electronics to manufacturing utilities, microwatts to world third largest, and touch screen techniques to parabolic dish communication systems. A power source, by description, is a device which converts an ac voltage line's outcome to a steady $\mathrm{dc}$ outcome or output stage. The ac voltage is converted first to create a pulsing dc, then sifted to make a seamless voltage. Eventually, regardless of changes in the ac line voltage as well as circuit trying to load, the voltage is controlled to generate a constant quantity produced.

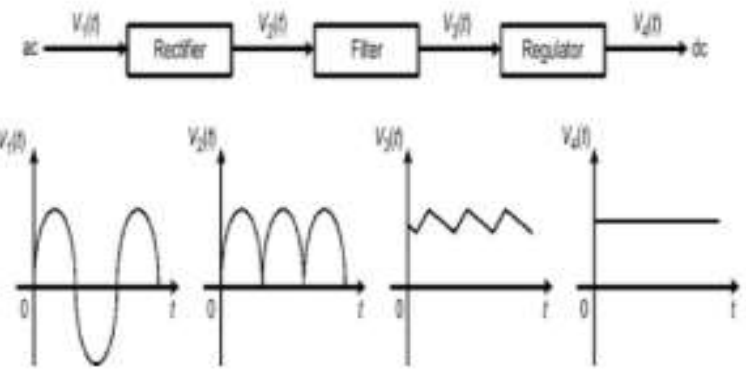

Figure 1 Basic Schematic of Power Supply

In a power source, the practices of correction, filtration, and regulatory oversight.

Distributed generation are gaining popularity as a way to reduce load expansion, incorporate occasional renewable, and avoid protracted power failures. A open construction or college, as well as a society power wall that supports different structures with shared ownership, are the two types of distributed generation. The owner/operator of a public micro grid appears to face greater challenges. The aggregation site is the person in charge of ensuring that these distributed generation are wellplanned and run efficiently. As a result, substations should be able to control loads and utility grid (DERs) within each constructing. When residential buildings are included in a society power system, the problem worsens. The aggregation site in a society power system should have clear visibility of residences loads and DERs in monitoring this sector's lots and lots.

Micro grids (MGs) are small self-contained propulsions that can function when the larger matrix is down, supply electricity in remote locations and in the aftermath of disasters. The implementation of distributed generation (DER) and the interaction assimilation of these assets with the MG management system or an MG controller were the focus of the majority of MG protest projects in the world. The assimilation of transformer 
processes was common in many of these initiatives, Solar photovoltaic (PV) and storage technologies, as well as no inverter-based structures such as fuel or fossil fuels generator and district heating units of various sizes and producers, are examples. Communications and physical world to memorandum between the DER and the MG control system are required in MG deployments to ensure resilient and sustainable procedure of an MG.

\section{Literature REVIEW}

(Chauhan \& Saini, 2014) [1] Economically unviable grid connection has resulted in the generating electricity at the end user clinic, which has been shown to be cost productive and useful to some extent. With a greater emphasis on cleaner technologies, renewable energies such as micro hydro, air, photovoltaic, biomass, and biogas are being investigated. This paper offers a comprehensive examination of a number of issues concerning the generation of electricity using an Incorporated Wind power Electricity Grid (IRES). For energy flow planning, concerns such as assimilation setups, additional storage, dimensioning methods, and control systems are thoroughly discussed Inclusion of renewable resources is analyzed in detail for hold applications using DC coupled, AC coupled, or hybrid DC-AC coupled setups. Energy storage choices for system components can be chosen depends on the length of volume needed in remote areas. The dynamical essence of source supply and availability at site accounts for the unknowns involved in developing an efficient IRES-based power generation system for remote areas. The different methods for sizing entire system that have been used and reported are proffered. In IRES, distributed control, centralized control, and combination control strategies for flow of energy planning were discussed.

(Fadaee \& Radzi, 2012) [2] For remote locations, an integration of renewable technology has been implemented as a green and reliability of power scheme. The use of hybrid energy units is steadily increasing, necessitating the solution of optimization for this system. Researchers were interested in using multi-objective optimization techniques to solve this problem in past years. As a result, an overall view of imposed multi-objective methodologies for hybrid power system utilizing optimization techniques was proposed in this research to aid ongoing and prospective research efforts. The findings show that there have been a few studies on using these methodologies to optimize many artifacts in a hybrid model, with the evolutionary algorithms and evolutionary algorithms being the most widely used methods. All rights belong, 2012 Elsevier Ltd.

(Fathima \& Palanisamy, 2015) [3] The electricity crisis has resulted from the rapid depletion of fossil fuels and the growing awareness of the importance of pollution prevention. As a result, researchers are working to find a new way to retrieve renewable power. With renewable energies, 'Micro grids' with Generating Units (DG) are being implemented. Optimization techniques justify the expense of a power system by allowing for cost- effective and reliable resource utilization. This paper aims to introduce the notion of hybrid power system (HRES) as well as state-of-the-art optimal control tools and methods for dc micro grid. incorporating renewable energy sources A structure of diverse goals has been underlined for which system approach have been decided to apply to embolden the micro grid, based on an extensive literature survey on HRES. There is also an overview of modeling work and apps of clean energy power storage sources.

(Ramli et al., 2018) [4]Micro - grids processes, such as photovoltaic panels (PV) strength and renewable turbines combined with generating units, are showing promise forms of energy that are financially viable for ongoing and prospective use in the face of rising energy requirements and the depletion of traditional fossil fuels. To ascertain cost of the system and serviceability, it is critical to optimize the size of hybrid power system (HMS) elements, which include stockpiling. The Inter Self-Adaptive Differential Evolution (Moseyed) algorithm is used to size an optimal PV/wind/diesel HMS with battery bank for the city of Istanbul in this article. Saudi Arabia is a country in the Middle East. The goals are unitary and autonomously using the inter optimizer, resulting in a reduction in computing time. The control strategy, which is needed to organize the various units that make up the HMS, is one of the most important factors to consider when trying to design and optimizing the HMS. The inter optimal solution is then used to investigate the Voltage spikes Stockpile Possibility (LPSP), Cost of Energy (COE), and Clean energy Factor (RF) in regards to HMS cost and durability, with three cases involving different house figures. The results show that it can be used to improve the HMS and put into practice. Furthermore, The outcomes of the enhancement using the proposed technique yielded a set of design solutions for the HMS, which will aid scholars and clinicians in selecting the best HMS configuration. Furthermore, selecting showed satisfactory results HMS elements is critical to ensuring that all load requirements are fulfilled with the least quantity of electricity and the highest degree of precision.

(Bilal et al., 2020) [5Discrepancy Evolution (DE) has become one of the most popular algorithms for multiobjective optimization since its founding in 1995. Because of its adaptability and versatility, DE has spawned a slew of customized variations for a range of sample and problem instances. The current research looks back over DE's nearly 25year history. The journey of DE clearly displays its fundamental parts like require increased, mutagenesis schemes, crossing schemes, variability in variables and hybridized variants, as well as different effective software of DE, in this extensive survey, which covers 283 research papers. This research also includes some key bibliometric markers, such as widely cited papers with more than 500 references, publishing trends since 1996, journal citations, and so on. The main goal of this proposal is to start serving as an elongated summary of DE's 25-year history, which will be distributed to relevant parties. The current survey is 
anticipated to catch the attention of newbie's in DE's ideology, as well as provide guidance to encounter scientists.

(Zahraee et al., 2016) [6Many governments have developed tremendous energy policies that promote photovoltaic systems in response to increased awareness of the need to reduce our unnatural weather fluctuations and the crucial growth in the price of traditional energy sources. Solar, wind, and water resources, for instance, are renewable resources that are both environmentally beneficial and have the biggest potential application. By combining renewable sources with standby unit to create a stable scheme, all of the load need circumstances can supply more inexpensive power, as well as ecologically sustainable resources, in contrast to single consumption. One of the most significant aspects of this kind of hybrid model, which might cover all of the load current with a small cash commitment, is designing the hybrid model pieces optimally. Despite the fact that a majority of researches have been conducted on the enhancement and dimensioning of hybrid systems, this research provides a comprehensive review of Artificial Intelligence optimized proposals in the literary works, contributing to the understanding of renewable facets for enhancing the systems' economic performance.

(Upadhyay \& Sharma, 2014) [7]The Energy consumption is expanding at a rapid rate, and traditional energy sources can meet it totally due to their finite supply. Furthermore, they have a significant environmental impact. In contrast, hybrid power system are a suitable blend of sustainable and quasi energy systems that takes into consideration the benefits of both, allowing for lower system implementation costs, as well as lower emissions levels. Improvements in total system stability, etc. The formulation and construction of a hybrid power system for a specific location is difficult due to the input variables of the resources examined changing arbitrarily over time and being independently of load needs. The paper contains a study of numerous critical sectors that must be taken into account while developing and implementing a hybrid method, such as topologies, criterion choice, size approaches, and controls and environmental generation. This will assist the architect in employing appropriate design limitations for designing hybrid power systems in grid linked or off network mode, depending on the needs of the locale. Elsevier Ltd., 2014.

(Bhandari et al., 2014) [8] The electricity consumption is growing at a rapid rate, and traditional energy sources cannot provide it totally due to its limited supply. Furthermore, they have a significant environmental impact. In contrast, hybrid power solutions are a corresponds of sustainable and quasi power systems that takes into consideration the benefits of both systems, allowing for lower system operational and maintenance costs, lower emissions levels, significantly improved reliability, and so on. The formulation and construction of a hybrid power system for a specific location is difficult due to the input variables of the resources examined varying randomly over time and being dependent of load needs. The paper contains a study of numerous critical sectors that must be taken into account while developing and implementing a micro grid, such as topologies, criterion choice, size approaches, and controls and power system. This will assist the designer in employing appropriate design limitations for designing hybrid power systems in grid linked or off network mode, depending on the needs of the locale.

\section{HYBRID ENERGY SYSTEM}

Hybrid power sources are still in their early stages of development. Technologies is projected to keep advancing in the near, allowing for greater applicability and cheaper prices. There'll be more standardized designs, making it easy to choose a system that is best suited to certain needs. There will be even more interaction here between multiple elements. Management, tracking, and diagnosis will be easier. Lastly, power electronics will be used more frequently. Several hybrid vehicles are already using thyristors, and as prices and dependability increase, they are likely to become increasingly common.

In comparison to a system based on a particular source, hybrid power system system (HESs) combine diverse generating, storing, and consuming techniques in an unified system, boosting overall advantages. Initially envisioned to incorporate traditional, exhaustible creation (e.g., diesel generators) with battery storage (BESSs), their meaning has now been enlarged to also include processes that are entirely powered by renewable power [e.g., solar photovoltaics (PV) and wind], or structures that merge distinct energy storage devices (e.g., BESSs, fuel cells, and supercapacitors). HESs have also evolved in capacity, moving from small, off-grid installations of a few kilowatts, normally designed for low voltage DC and AC, to bigger mega installations that can now link to the grid at voltage level.

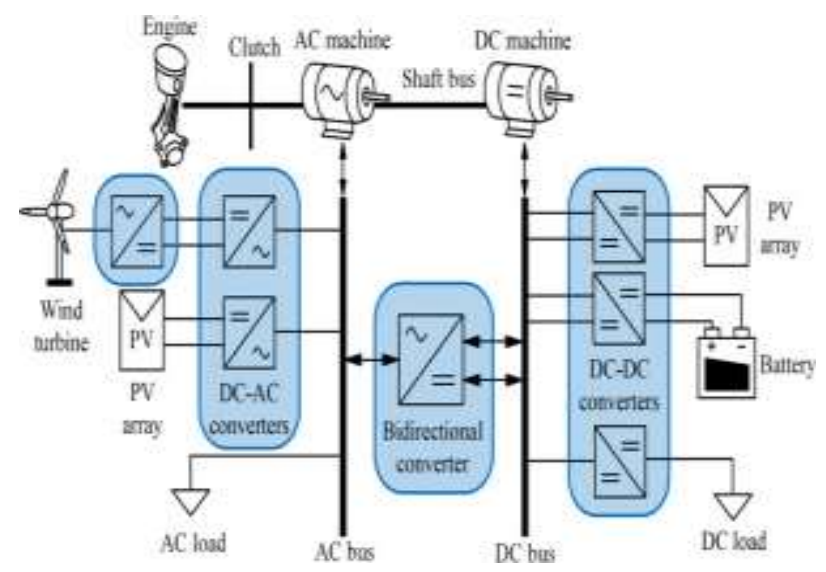

Figure 2 Hybrid Energy System

Among the major reasons that dictate the needs for the inverters used in HESs are the particular use cases for HESs, amount of funds, voltage regulation levels, and grid connectivity. In hang operations, hybrid energy systems are a very promising and long-term suitable for power production. In addition to purely 
cost-cutting objectives, it is becoming increasingly vital to examine other important considerations such as carbon reduction or network dependability enhancement. The creation and deployment of trustworthy and readily available multiple objective optimization tools, such as computer programs, will be aided by more study into the construction of general statistical equations. The creation of a cliched, validated, and comprehensive research methods for the formulation and layout of hybrid power devices - integrating planning and standardised designs to consider the qualities of the place, the adequate hybrid electricity grid, and all prospective operational situations for its proposal - would greatly enhance the adoption of these technologies. The use of a computer-aided process development technique could be very useful in this regard.

\section{DIFFERENTIAL EVOLUTIONARY ALGORITHM}

EAs, which are based on Natural selection and evolution, are very well for their knowledge to fix complex and complicated optimization issues. The major benefit of EAs over other numerical techniques is that they only need the objective functions and don't require qualities like separability or continuation. The effectiveness of an EA, on the other hand, is determined by the encoding, evolving operations, and parameterization such as slight variation and population numbers. Choosing appropriate model parameters is a problemdependent activity that usually necessitates a moment court hearing approach. If the adaptive method is necessary in an automated system or if the user lacks skill with the art of control input tuning, this method is not acceptable. Different parameter adaption strategies have been proposed to solve this. There are three types of variable blended learning: predictable, adaptation, and ego. Predetermined rules change the settings based on predefined logics without taking into account any input from the initial search.

The variable adaption is guided by adaptable rules, which integrate input from the search phaseEgo rules store framework of relevant into people and develop them alongside the encoded solutions. The best parameter values create good children who are more likely to survive and pass on the optimal parameter values. Feels a sense rules, in general, are those that primarily rely on input from the search process to drive parameter updates. Researchers suggest an ensembles method for the research, in which each variable has a pools of value vying to generate future offspring depending on their results in previous rounds. Even though the ensembles notion is universal and may be used with any based on genetic algorithm architecture, we use the heuristic algorithm (DE) method to illustrate it. DE, as suggested by Storn and Price, is a quick and easy method that utilizes effectively on a wide range of problems.

$\mathrm{DE}$ is an intrinsically concurrent inhabitants random search strategy. Engineering, communications, optical pattern matching, data processing, and power grids are just a few of the disciplines where DE has been proved useful. The DE individual's achievement is affected by the mutation strategic plan and limits on government such as overall population (NP), center frequency (CR), and scale factor (F). For various optimization and the same functions with varying criteria for consuming time of the system, the ideal values for the controller parameters can be varying. As a result, in able to fix a specific optimal solution effectively, it's usually required to conduct a moment trial-and-error look for the perfect approach and modify its related model parameters. However, a trial-and-error search procedure like this has a lot of computing overhead. Various methodologies with sets of conditions may be more successful than others when the populace of DE evolves across different areas in the search area.

\section{CONCLUSION}

The desire for energy is growing at a rapid rate, and conventional energy mechanisms cannot meet it completely due to the inadequate supply. Furthermore, they get a significant environmental impact. Throughout comparing, hybrid energy processes are a suitable combination of renewable and nonrenewable energy technologies that considers the benefits of both systems, allowing them to achieve greater efficiency. To accomplish a lowering in system operational and maintenance costs, lower exhaust levels, and enhance organizational system reliability The creation and analysis of a hybrid power system for a specific location is difficult due to the input variables of the source materials regarded differing randomly over time and being self - reliant of load demand. This paper provides the general review of hybrid energy systems and use of differential evolutionary algorithm

\section{REFERENCES}

[1] Chauhan, A., \& Saini, R. P. (2014). A review on Integrated Renewable Energy System based power generation for stand-alone applications: Configurations, storage options, sizing methodologies and control. Renewable and Sustainable Energy Reviews, 38, 99-120. https://doi.org/10.1016/j.rser.2014.05.079

[2] Fadaee, M., \& Radzi, M. A. M. (2012). Multi-objective optimization of a stand-alone hybrid renewable energy system by using evolutionary algorithms: A review. Renewable and Sustainable Energy Reviews, 16(5), 33643369. https://doi.org/10.1016/j.rser.2012.02.071

[3] Fathima, A. H., \& Palanisamy, K. (2015). Optimization in microgrids with hybrid energy systems - A review. Renewable and Sustainable Energy Reviews, 45, 431-446. https://doi.org/10.1016/j.rser.2015.01.059

[4] Ramli, M. A. M., Bouchekara, H. R. E. H., \& Alghamdi, A. S. (2018). Optimal sizing of PV/wind/diesel hybrid microgrid system using multi-objective self-adaptive differential evolution algorithm. Renewable Energy, 121, 400-411. https://doi.org/10.1016/j.renene.2018.01.058

[5] Bilal, Pant, M., Zaheer, H., Garcia-Hernandez, L., \& Abraham, A. (2020). Differential Evolution: A review of more than two decades of research. Engineering 
Applications of Artificial Intelligence, 90(January), 103479. https://doi.org/10.1016/j.engappai.2020.103479

[6] Zahraee, S. M., Khalaji Assadi, M., \& Saidur, R. (2016). Application of Artificial Intelligence Methods for Hybrid Energy System Optimization. Renewable and Sustainable Energy Reviews, 66, 617-630. https://doi.org/10.1016/j.rser.2016.08.028

[7] Upadhyay, S., \& Sharma, M. P. (2014). A review on configurations, control and sizing methodologies of hybrid energy systems. Renewable and Sustainable Energy Reviews, 38, 47-63. https://doi.org/10.1016/j.rser.2014.05.057

[8] Bhandari, B., Poudel, S. R., Lee, K. T., \& Ahn, S. H. (2014). Mathematical modeling of hybrid renewable energy system: A review on small hydro-solar-wind power generation. International Journal of Precision Engineering and Manufacturing - Green Technology, 1(2), 157-173. https://doi.org/10.1007/s40684-014-0021-4 